\title{
An insight into the mechanical properties of selected commercial oil and alkyd paint films containing cobalt blue
}

\author{
Laura Fuster-López ${ }^{\mathrm{a}, *}$, Francesca Caterina Izzo $^{\mathrm{b}}$, Valentina Damato ${ }^{\mathrm{b}}$, \\ Dolores J. Yusà-Marco ${ }^{\mathrm{a}}$, Elisabetta Zendri ${ }^{\mathrm{b}}$ \\ a Universitat Politècnica de València, Instituto Universitario de Restauración del Patrimonio, Camino de Vera s/n, 46022 Valencia, Spain

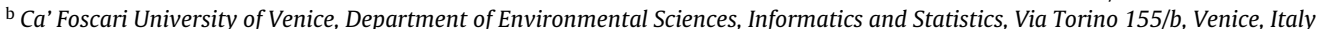

\section{A R T I C L E I N F O}

\section{Article history:}

Received 6 February 2018

Received in revised form

18 December 2018

Accepted 18 December 2018

Available online $\mathrm{xxx}$

\section{Keywords:}

Modern oil paints

Commercial formulations

Cobalt blue

Driers

Mechanical properties

GC-MS

\begin{abstract}
A B S T R A C T
Pigments and binders are known to influence drying and ageing mechanisms of paint films. However, the study of the long-term mechanical behaviour of paint films and its relationship with paint degradation and conservation still needs further investigation. This paper presents an insight into the role of cobalt blue pigment in the mechanical properties of some modern paint films by studying selected commercial oil and alkyd paint films containing cobalt blue. In addition, several representative mock-ups containing cobalt blue mixed with drying and semi-drying oils and some common additives were also tested for comparison purposes. Optical microscopy, SEM-EDX, XRF, VIS-reflectance spectroscopy, FTIR-ATR and GC-MS analysis were carried out to identify pigments, binders, additives and fillers. Uniaxial tensile measurements were run to test the mechanical performance of the studied paint films. The differences in the mechanical behaviour led to the evaluation of the discrepancies found in the chemical and physical properties of the different formulations studied.
\end{abstract}

(C) 2018 Elsevier Masson SAS. All rights reserved.

\section{Cobalt blue}

\subsection{Introduction}

The presence of wrinkles, cracks, flaking and delamination is usually registered in modern and contemporary oil and alkyd paintings. They are usually the consequence of complex failure mechanisms as a result of chemical degradation processes [1-6] and changes in the mechanical and dimensional properties of the paint film over time and where the environment does not necessarily play a particular role [7-10].

Previous studies have shown a correlation between the different composition, drying and aging of lipidic binding media and the changes in the mechanical properties in paint films over time. The mechanical properties may also be modified by the ionic bonds formed by pigments with the cross-linked network of the medium. These changes are related to the on-going chemical processes that oil paint films experience. In general, oil paint films become stiffer over time which is an indication of the progressive increase of modulus of elasticity (E) and ultimate tensile strength (UTS), and a

\footnotetext{
* Corresponding author.

E-mail address: laufuslo@crbc.upv.es (L. Fuster-López).
}

decrease in the strain to failure or, in other words, with a decreasing ability to elongate before breaking [11-13]. Pigments govern such changes and their magnitude but also determine how early such changes will take place in the drying process of a given paint film $[14,15]$. For example, some pigments can react with the free fatty acids and various compounds formed during oxidation and may inhibit the diffusion of oxygen in the paint film as well as the evaporation of volatile compounds from the film [13]. Pigments made from reactive metals act as catalysts, which accelerate drying. This is the case, for instance, of cobalt-based pigment.

Cobalt Blue pigment, known as Pigment PB 28, is a cobalt (II) oxide-aluminium oxide, or cobalt (II) aluminate, $\mathrm{CoAl}_{2} \mathrm{O}_{4}$ made by sintering cobalt (II) oxide with alumina at temperatures above $1200^{\circ} \mathrm{C}$. Cobalt blue was synthesized by Thénard in 1803 and immediately manufactured on a commercial scale; it rapidly became one the most common blue pigments used in modern art $[16,17]$, despite being relatively expensive. This could explain eventual cases of pigment adulteration (either with organic or inorganic pigments) throughout history such as the mixture with artificial ultramarine (PB 29, $\mathrm{Na}_{8-10} \mathrm{Al}_{6} \mathrm{Si}_{6} \mathrm{O}_{2-4} \mathrm{~S}_{2-4}$ ) [18]. Cobalt blue pigment is also a surface siccative. It is the most commonly used primary drier and the most active. It acts as an oxidation catalyst since its exhibits more than one oxidation state. As other primary driers, cobalt blue pigment catalyzes cross-linking of the medium and - 
Table 1

Commercial oil and alkyd paint samples studied (from Mecklenburg's Paint Reference Collection).

\begin{tabular}{|c|c|c|c|c|c|c|}
\hline Sample & & & Thickness & Casting date & $\begin{array}{l}\text { Tensile tests run (different } \\
\text { samples cut from the same } \\
\text { piece of cast paint) in }\end{array}$ & Observations \\
\hline GR-CB-ARLO & $\begin{array}{l}\text { Cobalt blue, } \\
\text { PB28 }\end{array}$ & $\begin{array}{l}\text { Alkali refined } \\
\text { linseed oil }\end{array}$ & $250 \mu \mathrm{m}$ & 1999 & $\begin{array}{l}2001 \text { (Mecklenburg et al., } \\
2013 \text { ) [11] } \\
2017 \text { (this study) }\end{array}$ & \\
\hline GA-CB-ARLO & $\begin{array}{l}\text { Cobalt blue, } \\
\text { PB28 }\end{array}$ & $\begin{array}{l}\text { Alkali refined } \\
\text { linseed oil }\end{array}$ & $250 \mu \mathrm{m}$ & 1999 & $\begin{array}{l}2001 \text { (Mecklenburg et al., } \\
2013 \text { ) [11] } \\
2017 \text { (this study) }\end{array}$ & \\
\hline GA-CB-LIT & $\begin{array}{l}\text { Cobalt blue, } \\
\text { PB28 }\end{array}$ & Linseed oil & $230 \mu \mathrm{m}$ & 1999 & $\begin{array}{l}2001 \text { (Mecklenburg et al., } \\
2013 \text { ) [11] } \\
2017 \text { (this study) }\end{array}$ & $\begin{array}{l}\text { Significant } \\
\text { wrinkles observed } \\
\text { on the surface } \\
\text { Darker tone }\end{array}$ \\
\hline $\mathrm{WN}-\mathrm{CB}$ & $\begin{array}{l}\text { Cobalt blue, } \\
\text { PB28 }\end{array}$ & $\begin{array}{l}\text { Linseed/safflowe } \\
\text { oils }\end{array}$ & $\mathrm{B} 40 \mu \mathrm{m}$ & 1998 & $\begin{array}{l}2001 \text { (Mecklenburg et al., } \\
\text { 2013) [11] }\end{array}$ & $\begin{array}{l}\text { Too sticky } \\
\text { It was not possible } \\
\text { to test it in } 2017\end{array}$ \\
\hline WN-ALK & $\begin{array}{l}\text { Cobalt blue, } \\
\text { PB28 }\end{array}$ & Alkyd resin & $200 \mu \mathrm{m}$ & 1998 & $\begin{array}{l}2001 \text { (Mecklenburg et al., } \\
2013 \text { ) [11] } \\
2017 \text { (this study) }\end{array}$ & $\begin{array}{l}\text { Microprotuberances } \\
\text { on the surface }\end{array}$ \\
\hline WN-GR-ALK & $\begin{array}{l}\text { Cobalt blue, } \\
\text { PB28 }\end{array}$ & Alkyd & $160 \mu \mathrm{m}$ & 1999 & $\begin{array}{l}2001 \text { (Mecklenburg et al., } \\
2013 \text { ) [11] } \\
2017 \text { (this study) }\end{array}$ & \\
\hline
\end{tabular}

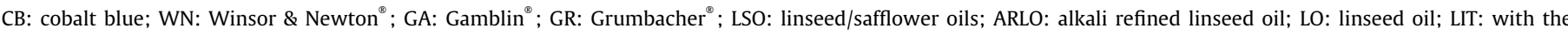
commercial addition of Litharge.

a Based on manufacturers' datasheets available in the 1980-1990's.

within this process- hydroxyl, carbonyl and carboxylic groups are formed accelerating the drying of the surface.

This double role of $\mathrm{CoAl}_{2} \mathrm{O}_{4}$ (as a pigment and a siccative) has evident implications in the conservation practice, in particular in the formation of superficial wrinkles. The presence of surface wrinkles in paint films has traditionally been explained as a consequence of the plastic flow experienced by the paint film due to the excess of binder and low pigment volume concentration (PVC), and therefore usually associated to long drying times. Nevertheless, there are other sources for the wrinkled appearance of cobalt blue paint films such as its siccative nature. Being cobalt blue a surface siccative, it tends to create an initial surface film that slows down the diffusion of oxygen in the bulk of the paint, leading often to a distinctive effect in the form of wrinkles (see figures in Tables 1 and 2). In this latter case, wrinkles occur naturally as a consequence of pigment-binder interaction during the drying process and where PVC not necessarily has any influence. Both mechanisms are challenging but require a different approach in day-to-day conservation practice.

\subsection{Aim of this study}

In this paper, the mechanical properties of selected commercial oil and alkyd paints containing cobalt blue pigment are studied. For this purpose, manufactured formulations and custom-made paint reconstructions were prepared. The aim of this study was to assess the different mechanical behaviour of the paint films tested and to gain an insight into complex paint formulations. The influence of some primary factors such as the amount and type of pigment and binder, the presence and type of additives as well as the hydrolysis mechanisms experienced may have in the long-term mechanical behaviour of the paint films studied is discussed.

\section{Materials and methods}

\subsection{Paint samples and sample preparation}

In this research, three different sets of cobalt blue paint films were studied.

We selected four cobalt blue oil samples manufactured by Grumbacher, Gamblin and Winsor \& Newton (W\&N) and two cobalt blue alkyd oil paint films from W\&N belonging to the Mecklenburg's Paint Reference Collection at the Smithsonian Museum Conservation Institute (USA). The oil films tested and discussed in this paper were prepared late in the 1990's on polyester film and allowed to dry in controlled environmental conditions since then (Table 1).

A second set of samples consists of four commercial cobalt blue oil paints manufactured by Titan, W\&N, Talens-Van Gogh and Maimeri as reported in Table 2. The paints were purchased and cast in 2014 on polyester film and allowed to dry in controlled environmental conditions.

The third set of samples consists of five custom-made paint film reconstructions, (Table 3). 1L was prepared mixing cobalt blue PB28 and cold pressed linseed oil (CPLO) from Kremer. 2L, 3L and 4L were prepared similarly but with the progressive incorporation of additives: $25 \%$ of the total amount of pigment were inorganic fillers (mixture of calcite, gypsum and kaolin). Five percent aluminum stearate and $10 \%$ castor wax (i.e. hydrogenated castor oil purchased from Sigma-Aldrich) were also added to gain an insight into the effects specific additives can induce in the mechanical properties of a given cobalt blue oil paint film. The paint film labelled as $5 \mathrm{~F}$ was prepared by mixing CPLO with sunflower and safflower oils (Sigma-Aldrich) together with all the above-mentioned additives. This formulation is consistent with the complex mixtures of organic 
Table 2

Commercial oil paint samples studied (2014).

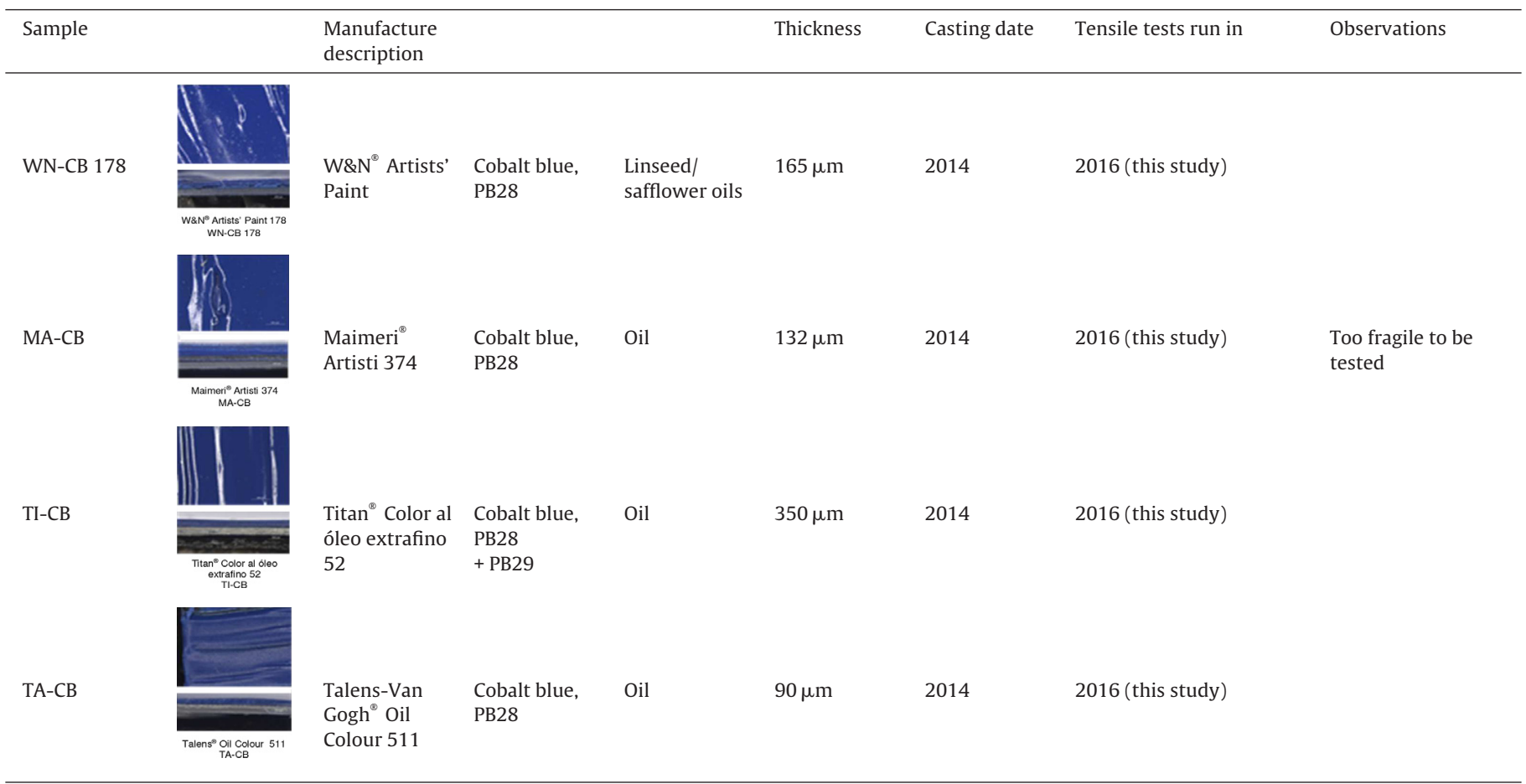

Table 3

Representative custom-made cobalt oil paint films (2014).

\begin{tabular}{|c|c|c|c|c|c|c|}
\hline Sample & Description & & & Thickness & Casting date & Tensile tests run in \\
\hline 1.L & $\begin{array}{l}\text { CPLO } \\
(54.5 \%)\end{array}$ & $\begin{array}{l}\text { Cobalt blue PB28 } \\
(45.5 \%)\end{array}$ & No additive & $250 \mu \mathrm{m}$ & 2014 & 2017 (this study) \\
\hline 2.L & $\begin{array}{l}\text { CPLO } \\
(50 \%)\end{array}$ & $\begin{array}{l}\text { Cobalt blue PB28 } \\
(50 \%)\end{array}$ & $\begin{array}{l}\mathrm{CaCO}_{3}+\mathrm{CaSO} \\
\text { of pigment })\end{array}$ & $150 \mu \mathrm{m}$ & 2014 & 2017 (This study) \\
\hline 3.L & $\begin{array}{l}\text { CPLO } \\
(50 \%)\end{array}$ & $\begin{array}{l}\text { Cobalt blue PB28 } \\
(50 \%)\end{array}$ & $\begin{array}{l}\mathrm{CaCO}_{3}+\mathrm{CaSO}_{4}+2 \mathrm{SiO}_{2} \cdot \mathrm{Al}_{2} \mathrm{O}_{3} \cdot 2\left(\mathrm{OH}_{2}\right)(25 \% \\
\text { of pigment })+\left[\mathrm{CH}_{3}\left(\mathrm{CH}_{2}\right)_{16} \mathrm{COO}\right]_{3} \mathrm{Al}(5 \% \text { of } \\
\text { pigment })\end{array}$ & $140 \mu \mathrm{m}$ & 2014 & 2017 (this study) \\
\hline 4.L & $\begin{array}{l}\text { CPLO } \\
(50 \%)\end{array}$ & $\begin{array}{l}\text { Cobalt blue PB28 } \\
(50 \%)\end{array}$ & $\begin{array}{l}\mathrm{CaCO}_{3}+\mathrm{CaSO}_{4}+2 \mathrm{SiO}_{2} \cdot \mathrm{Al}_{2} \mathrm{O}_{3} \cdot 2\left(\mathrm{OH}_{2}\right)(25 \% \\
\text { of pigment })+\left[\mathrm{CH}_{3}\left(\mathrm{CH}_{2}\right)_{16} \mathrm{COO}\right]_{3} \mathrm{Al}(5 \% \text { of } \\
\text { pigment })+ \text { castor wax ( } 10 \% \text { of pigment })\end{array}$ & $110 \mu \mathrm{m}$ & 2014 & 2017 (this study) \\
\hline 5. F & $\begin{array}{l}\text { CPLO + } \\
\text { safflower + } \\
\text { sunflower } \\
(50 \%)\end{array}$ & $\begin{array}{l}\text { Cobalt blue PB28 } \\
(50 \%)\end{array}$ & $\begin{array}{l}\mathrm{CaCO}_{3}+\mathrm{CaSO}_{4}+2 \mathrm{SiO}_{2} \cdot \mathrm{Al}_{2} \mathrm{O}_{3} \cdot 2\left(\mathrm{OH}_{2}\right)(25 \% \\
\text { of pigment })+\left[\mathrm{CH}_{3}\left(\mathrm{CH}_{2}\right)_{16} \mathrm{COO}\right]_{3} \mathrm{Al}(5 \% \text { of } \\
\text { pigment })+ \text { castor wax ( } 5 \% \text { of pigment })\end{array}$ & $190 \mu \mathrm{m}$ & 2014 & 2017 (this study) \\
\hline
\end{tabular}

and inorganic compounds that can be found in manufactured paints $[3,19]$.

\subsection{Analytical techniques and methods}

The selected paint films were studied by the joint use of several analytical techniques to obtain data about their composition, morphology and mechanical behaviour.

Tensile tests were conducted using custom built miniature tensile testers [21]. Average sample measurements were $6.5 \mathrm{~mm}$ (width) $\times 0.30 \mathrm{~mm}$ (thickness) and $7.5 \mathrm{~mm}$ (gauge length). Average thickness and width were measured three times along the gauge length with both a Mitutoyo 103-137 micrometer and a digital calliper gauge micrometer. Two samples from the same paint strips cast in the 1990s and tested by Mecklenburg in the very early stages of their drying process $[20,21]$ were tested for this study thus allowing the monitoring of changes induced after having been drying for 18 years in a controlled environment. For this purpose, the polyester film was removed and the tests were run on free unsupported paint films. The samples were clamped in the testing gauges and allowed to come into equilibrium in the chamber for $48 \mathrm{~h}$ at $50 \pm 0.5 \% \mathrm{RH}$ and $23 \pm 0.5^{\circ} \mathrm{C}$ prior to testing. A Testo $608-\mathrm{H} 2$ thermohygrometer together with a precision hygrometer (Mitchell Optidew Vision High Performance Optical Dew-Point Transmitter) monitored relative humidity and temperature in the chamber. All the tests were conducted at the same strain rate. Increments of $0.0625 \mathrm{~mm}$ strain were applied progressively at 30 -second intervals. Test were performed at $3.75 \mathrm{~mm} / \mathrm{min}$ speed. Load-displacement data were converted to stress and strain values using a customized Microsoft Excel spreadsheet.

Microsamples of free films were taken from the dried paints and observed with a Leica DMR microscope in reflected light $(25 \times$ to $400 \times$ magnification).

A Jeol JSM 6300 scanning electron microscope operating with a Link-Oxford-Isis energy-dispersive X-ray microanalysis system was used to observe surface and cross sections of the films (20 kV accelerating voltage, 2.10-9 A beam current and $15 \mathrm{~mm}$ distance). Elemental semi-quantitative weight percentages were calculated by EDX probe according to ZAF matrix correction [22-24]. 
Table 4

Resume of the results of the chemical characterization of the commercial oil and alkyd paint samples studied (from Mecklenburg's Paint Reference Collection).

\begin{tabular}{|c|c|c|c|c|c|c|c|}
\hline Samples & $\begin{array}{l}\text { Optical } \\
\text { microscopy }\end{array}$ & $\begin{array}{l}\text { SEM } \\
\text { observations }\end{array}$ & EDX & VIS-reflectance & FTIR-ATR (D2nd) & GC-MS & Considerations \\
\hline GR-CB-AR LO & $\begin{array}{l}\text { Low roughness } \\
\text { Light blue } \\
\text { Cross-section: } \\
\text { compact film. } \\
\text { Dark tone on the } \\
\text { bottom and } \\
\text { lighter close to } \\
\text { the surface }\end{array}$ & $\begin{array}{l}\text { Fine } \\
\text { granulometry } \\
\text { with some coarse } \\
\text { grains } \\
\text { Organic material } \\
\text { concentrated on } \\
\text { the surface } \\
\text { Homogeneous } \\
\text { distribution of Al } \\
\text { and Co }\end{array}$ & $\begin{array}{l}\mathrm{Co}, \mathrm{Al}, \mathrm{Ca}, \mathrm{P}, \mathrm{Si} \text {, } \\
\mathrm{Mg} \\
\% \mathrm{Co}=21\end{array}$ & Cobalt blue & $\begin{array}{l}\text { Drying oil } \\
\text { Carboxylates and } \\
\text { free fatty acids } \\
\text { (stearates) } \\
\mathrm{CoAl}_{2} \mathrm{O}_{4} \\
\text { Silicates } \\
\text { Calcium sulphate }\end{array}$ & $\begin{array}{l}\text { Saturated monocarboxylic } \\
\text { (lauric, myristic, palmitic, } \\
\text { stearic) and dicarboxylic } \\
\text { (suberic, azelaic, sebacic) acids; } \\
\text { unsaturated (oleic) } \\
\text { Acids; glycerol } \\
\mathrm{P} / \mathrm{S}=0.8 \\
\mathrm{~A} / \mathrm{P}=2.5 \\
\mathrm{O} / \mathrm{S} \text { « } 0.1\end{array}$ & \\
\hline GA-CB-ARLO & $\begin{array}{l}\text { Plain and glossy } \\
\text { surface } \\
\text { Light blue } \\
\text { Cross-section: } \\
\text { compact film and } \\
\text { uniform tome }\end{array}$ & $\begin{array}{l}\text { Homogeneous } \\
\text { and compact } \\
\text { granulometry } \\
\text { (well grinded: } \\
1 \mu \mathrm{m} \text { ) } \\
\text { Uniform } \\
\text { distribution of Co } \\
\text { and Al particles }\end{array}$ & $\begin{array}{l}\mathrm{Co}, \mathrm{Al}, \mathrm{Ca}, \mathrm{Si} \\
\% \mathrm{Co}=24\end{array}$ & Cobalt blue & $\begin{array}{l}\text { Drying oil } \\
\text { Carboxylates and } \\
\text { free fatty acids } \\
\text { (calcium } \\
\text { sterate?) } \\
\text { Calcium } \\
\text { carbonate } \\
\text { Calcium sulphate } \\
\mathrm{CoAl}_{2} \mathrm{O}_{4}\end{array}$ & $\begin{array}{l}\text { Saturated monocarboxylic } \\
\text { (lauric, myristic, palmitic, } \\
\text { stearic) and dicarboxylic } \\
\text { (pimelic, suberic, azelaic, } \\
\text { sebacic) acids; unsaturated } \\
\text { (oleic) } \\
\text { Acids; glycerol } \\
\mathrm{P} / \mathrm{S}=1.1 \\
\mathrm{~A} / \mathrm{P}=2.3 \\
\mathrm{O} / \mathrm{S} \text { « } 0.1\end{array}$ & \\
\hline GA-CB-LIT & $\begin{array}{l}\text { Dark blue and } \\
\text { opaque surface } \\
\text { Wrinkles on the } \\
\text { surface } \\
\text { Cross-section: } \\
\text { heterogeneous } \\
\text { tone (lighter in } \\
\text { the middle) }\end{array}$ & $\begin{array}{l}\text { Homogeneous } \\
\text { and fine } \\
\text { granulometry: } \\
\text { uniform } \\
\text { distribution of Co } \\
\text { and Al particles } \\
+ \text { Pb particles }\end{array}$ & $\begin{array}{l}\mathrm{Co}, \mathrm{Al}, \mathrm{Pb}, \mathrm{S}, \mathrm{Fe}, \\
\mathrm{Ca} \\
\% \mathrm{Co}=14\end{array}$ & $\begin{array}{l}\text { Cobalt } \\
\text { blue }+ \text { litharge }\end{array}$ & $\begin{array}{l}\text { Drying oil (free } \\
\text { fatty acids and } \\
\text { Carboxylates) } \\
\mathrm{CaCO}_{3} \\
\mathrm{CoAl}_{2} \mathrm{O}_{4}\end{array}$ & $\begin{array}{l}\text { Saturated monocarboxylic } \\
\text { (lauric, myristic, palmitic, } \\
\text { stearic) and dicarboxylic } \\
\text { (pimelic, suberic, azelaic, } \\
\text { sebacic) acids; unsaturated } \\
\text { (oleic) } \\
\text { Acids; traces of glycerol } \\
\mathrm{P} / \mathrm{S}=1.6 \\
\mathrm{~A} / \mathrm{P}=4.2 \\
\mathrm{O} / \mathrm{S} « 0.1\end{array}$ & \\
\hline WN-CB & $\begin{array}{l}\text { Homogeneous } \\
\text { surface } \\
\text { Light blue } \\
\text { Cross-section: } \\
\text { heterogeneous } \\
\text { tone }\end{array}$ & $\begin{array}{l}\text { Very } \\
\text { homogeneous } \\
\text { and fine } \\
\text { granulometry } \\
\text { Concentration of } \\
\text { organic material } \\
\text { Uniform } \\
\text { distribution of Co } \\
\text { and Al }\end{array}$ & $\begin{array}{l}\mathrm{Co}, \mathrm{Al}, \mathrm{Ca}, \mathrm{Mg}, \mathrm{Si} \text {, } \\
\mathrm{K}, \mathrm{S}, \mathrm{Fe} \\
\% \mathrm{Co}=18\end{array}$ & Cobalt blue & $\begin{array}{l}\text { Drying oil } \\
\text { Aluminium } \\
\text { stearate } \\
\text { (carboxylates) } \\
\mathrm{CoAl}_{2} \mathrm{O}_{4} \\
\text { Silicates } \\
\text { DOLOMITE }\end{array}$ & $\begin{array}{l}\text { Saturated monocarboxylic } \\
\text { (lauric, myristic, palmitic, } \\
\text { stearic) and dicarboxylic } \\
\text { (pimelic, suberic, azelaic, } \\
\text { sebacic) acids; unsaturated } \\
\text { (oleic) } \\
\text { Acids; glycerol; long-chain } \\
\text { monocarboxylic (behenic and } \\
\text { arachidic) acids } \\
\mathrm{P} / \mathrm{S}=2.8 \\
\mathrm{~A} / \mathrm{P}=2.1 \\
\mathrm{O} / \mathrm{S}=0.4\end{array}$ & \\
\hline WN-GR-ALK & $\begin{array}{l}\text { Plain and } \\
\text { homogeneous } \\
\text { surface } \\
\text { Medium-light } \\
\text { blue } \\
\text { Cross-section: } \\
\text { compact film }\end{array}$ & $\begin{array}{l}\text { Homogeneous } \\
\text { and fine } \\
\text { granulometry: } \\
\text { very compact } \\
\text { Uniform } \\
\text { distribution of Co } \\
\text { and Al }\end{array}$ & $\begin{array}{l}\mathrm{Co}, \mathrm{Al}, \mathrm{Ca}, \mathrm{Zn}, \mathrm{Si} \text {, } \\
\mathrm{K}, \mathrm{Mg}, \mathrm{Fe} \\
\% \mathrm{Co}=24\end{array}$ & $\begin{array}{l}\text { Cobalt } \\
\text { blue + white } \\
\text { pigment } \\
\text { (probably zinc } \\
\text { white) }\end{array}$ & $\begin{array}{l}\text { Alkyd resin, } \\
\text { carboxylates ( } \mathrm{Zn} \\
\text { soaps) } \\
\mathrm{CoAl}_{2} \mathrm{O}_{4} \\
\text { Silicates }\end{array}$ & $\begin{array}{l}\text { Saturated monocarboxylic } \\
\text { (palmitic, stearic) and } \\
\text { dicarboxylic (suberic, azelaic, } \\
\text { sebacic) acids; unsaturated } \\
\text { (oleic) } \\
\text { Acids; glycerol; } \\
\text { pentaerythritol; phtalic acid; } \\
\text { 12-hydroxy stearic acid } \\
\mathrm{P} / \mathrm{S}=1.6 \\
\mathrm{~A} / \mathrm{P}=1.9 \\
\mathrm{O} / \mathrm{S}=0.1\end{array}$ & \\
\hline WN-ALK & $\begin{array}{l}\text { Plain and } \\
\text { homogeneous } \\
\text { surface } \\
\text { Medium-light } \\
\text { blue } \\
\text { Cross-section: } \\
\text { compact draft } \\
\text { and } \\
\text { heterogeneous } \\
\text { tone for lighter } \\
\text { particles }\end{array}$ & $\begin{array}{l}\text { Presence of } \\
\text { surface cracking } \\
\text { Fine } \\
\text { granulometry } \\
\text { and uniform } \\
\text { distribution of Co } \\
\text { and Al }\end{array}$ & $\begin{array}{l}\mathrm{Co}, \mathrm{Al}, \mathrm{Ca}, \mathrm{Fe}, \mathrm{S}, \\
\mathrm{Mg}, \mathrm{Zn} \\
\% \mathrm{Co}=21\end{array}$ & $\begin{array}{l}\text { Cobalt } \\
\text { blue + white } \\
\text { pigment } \\
\text { (probably zinc } \\
\text { white) }\end{array}$ & $\begin{array}{l}\text { Alkyd resin } \\
\text { Carboxylates } \\
\text { (Stearate of } \\
\text { Aluminum) } \\
\mathrm{CoAl}_{2} \mathrm{O}_{4} \\
\text { Calcium and } \\
\text { magnesium } \\
\text { carbonates }\end{array}$ & $\begin{array}{l}\text { Saturated monocarboxylic } \\
\text { (palmitic, stearic) and } \\
\text { dicarboxylic (pimelic, suberic, } \\
\text { azelaic, sebacic) acids; } \\
\text { unsaturated (oleic) } \\
\text { Acids; glycerol; } \\
\text { pentaerythritol; phtalic acid; } \\
\text { 12-hydroxy stearic acid } \\
\mathrm{P} / \mathrm{S}=1.7 \\
\mathrm{~A} / \mathrm{P}=1.9 \\
\mathrm{O} / \mathrm{S} \text { « } 0.1\end{array}$ & \\
\hline WN-CB 178 & $\begin{array}{l}\text { Glossy and } \\
\text { wrinkled surface } \\
\text { Medium-dark } \\
\text { blue } \\
\text { Cross-section: } \\
\text { divided in two, } \\
\text { dark tone on the } \\
\text { surface }\end{array}$ & $\begin{array}{l}\text { Presence of } \\
\text { surface cracking } \\
\text { Fine } \\
\text { granulometry } \\
\text { and uniform } \\
\text { distribution of Co } \\
\text { and Al }\end{array}$ & $\begin{array}{l}\mathrm{Co}, \mathrm{Al}, \mathrm{Mg}, \mathrm{Cl}, \mathrm{Cr} \text {, } \\
\mathrm{K} \\
\% \mathrm{Co}=18\end{array}$ & Cobalt blue & $\begin{array}{l}\text { Drying oil } \\
\text { (partially } \\
\text { unsaturated) } \\
\text { Carboxylates } \\
\text { (aluminium } \\
\text { stearate) } \\
\mathrm{CoAl}_{2} \mathrm{O}_{4} \\
\text { Silicates } \\
\text { Calcium sulphate } \\
\text { Dolomite }\end{array}$ & $\begin{array}{l}\text { Saturated monocarboxylic } \\
\text { (lauric, myristic, palmitic, } \\
\text { stearic) and dicarboxylic } \\
\text { (suberic, azelaic, sebacic) acids; } \\
\text { unsaturated (oleic) } \\
\text { Acids; glycerol; long-chain } \\
\text { monocarboxylic (behenic and } \\
\text { arachidic) acids } \\
\mathrm{P} / \mathrm{S}=2.5 \\
\mathrm{~A} / \mathrm{P}=1.4 \\
\mathrm{O} / \mathrm{S}=1.3\end{array}$ & $\begin{array}{l}\text { Linseed oil and } \\
\text { safflower/sunflower }\end{array}$ \\
\hline
\end{tabular}


Table 4 (Continued)

\begin{tabular}{|c|c|c|c|c|c|c|c|}
\hline Samples & $\begin{array}{l}\text { Optical } \\
\text { microscopy }\end{array}$ & $\begin{array}{l}\text { SEM } \\
\text { observations }\end{array}$ & EDX & VIS-reflectance & FTIR-ATR (D2nd) & GC-MS & Considerations \\
\hline MA-CB & $\begin{array}{l}\text { Glossy and } \\
\text { wrinkled surface } \\
\text { Light blue } \\
\text { Cross-section: } \\
\text { stratified surface }\end{array}$ & $\begin{array}{l}\text { Fine } \\
\text { granulometry } \\
\text { and homogenous } \\
\text { Lighter particles } \\
\text { of additive } \\
\text { Co-concentred } \\
\text { on bulk and Al on } \\
\text { the surface }\end{array}$ & $\begin{array}{l}\mathrm{Al}, \mathrm{Co}, \mathrm{Si}, \mathrm{Na}, \mathrm{Zn}, \\
\mathrm{Cl} \\
\% \mathrm{Co}=24\end{array}$ & Cobalt blue & $\begin{array}{l}\text { Drying oil } \\
\text { (partially } \\
\text { unsaturated) } \\
\text { Carboxylates } \\
\text { (aluminium and } \\
\text { zinc stearate) } \\
\mathrm{CoAl}_{2} \mathrm{O}_{4} \\
\text { (Green } \\
\text { Phtalocianine?) } \\
\text { Silicates }\end{array}$ & $\begin{array}{l}\text { Saturated monocarboxylic } \\
\text { (lauric, myristic, palmitic, } \\
\text { stearic) and dicarboxylic } \\
\text { (suberic, azelaic, sebacic) acids; } \\
\text { unsaturated (oleic) } \\
\text { Acids; glycerol } \\
\mathrm{P} / \mathrm{S}=1.9 \\
\mathrm{~A} / \mathrm{P}=2.1 \\
\mathrm{O} / \mathrm{S}=0.5\end{array}$ & \\
\hline TI-CB & $\begin{array}{l}\text { Glossy surface } \\
\text { Medium-dark } \\
\text { tone } \\
\text { Cross-section: } \\
\text { compact and } \\
\text { homogeneous } \\
\text { draft }\end{array}$ & $\begin{array}{l}\text { Heterogeneous } \\
\text { granulometry } \\
\text { with bigger } \\
\text { particles of } \mathrm{Ca} \\
\text { Surface cracking } \\
\text { Uniform } \\
\text { distribution of Co } \\
\text { and } \mathrm{Al}\end{array}$ & $\begin{array}{l}\mathrm{Co}, \mathrm{Al}, \mathrm{Ca}, \mathrm{Si}, \mathrm{S}, \\
\mathrm{Na}, \mathrm{K}, \mathrm{Fe} \\
\% \mathrm{Co}=9\end{array}$ & $\begin{array}{l}\text { Cobalt } \\
\text { blue + ultramarine }\end{array}$ & $\begin{array}{l}\text { Drying oil } \\
\text { (partially } \\
\text { unsaturated) } \\
\text { Carboxylates } \\
\text { (aluminium } \\
\text { stearate) } \\
\mathrm{CoAl}_{2} \mathrm{O}_{4} \\
\text { Ultramarine blue } \\
\text { Calcium sulphate } \\
\text { Silicates } \\
\text { Calcium } \\
\text { carbonate }\end{array}$ & $\begin{array}{l}\text { Saturated monocarboxylic } \\
\text { (lauric, myristic, palmitic, } \\
\text { stearic) and dicarboxylic } \\
\text { (suberic, azelaic, sebacic) acids; } \\
\text { unsaturated (oleic) } \\
\text { Acids; glycerol } \\
\mathrm{P} / \mathrm{S}=1.8 \\
\mathrm{~A} / \mathrm{P}=1.4 \\
\mathrm{O} / \mathrm{S}=1.3\end{array}$ & \\
\hline $\mathrm{TA}-\mathrm{CB}$ & $\begin{array}{l}\text { Satin surface } \\
\text { Dark and } \\
\text { uniform tone } \\
\text { Cross-section: } \\
\text { heterogeneous } \\
\text { tone (lighter in } \\
\text { the middle) }\end{array}$ & $\begin{array}{l}\text { Homogeneous } \\
\text { granulometry } \\
\text { and superficial } \\
\text { cracking } \\
\text { Uniform } \\
\text { distribution of Co } \\
\text { and Al }\end{array}$ & $\begin{array}{l}\mathrm{Co}, \mathrm{Al}, \mathrm{Ca}, \mathrm{Zn}, \mathrm{Mg} \\
\% \mathrm{Co}=10\end{array}$ & Cobalt blue & $\begin{array}{l}\text { Drying oil } \\
\text { (partially } \\
\text { unsaturated) } \\
\text { Carboxylates } \\
\text { (zinc stearate) } \\
\mathrm{CoAl}_{2} \mathrm{O}_{4} \\
\text { Silicates } \\
\text { Calcium } \\
\text { carbonate }\end{array}$ & $\begin{array}{l}\text { Saturated monocarboxylic } \\
\text { (lauric, myristic, palmitic, } \\
\text { stearic) and dicarboxylic } \\
\text { (suberic, azelaic, sebacic) acids; } \\
\text { unsaturated (oleic) } \\
\text { Acids; glycerol } \\
\mathrm{P} / \mathrm{S}=0.9 \\
\mathrm{~A} / \mathrm{P}=0.7 \\
\mathrm{O} / \mathrm{S}=1.8\end{array}$ & \\
\hline
\end{tabular}
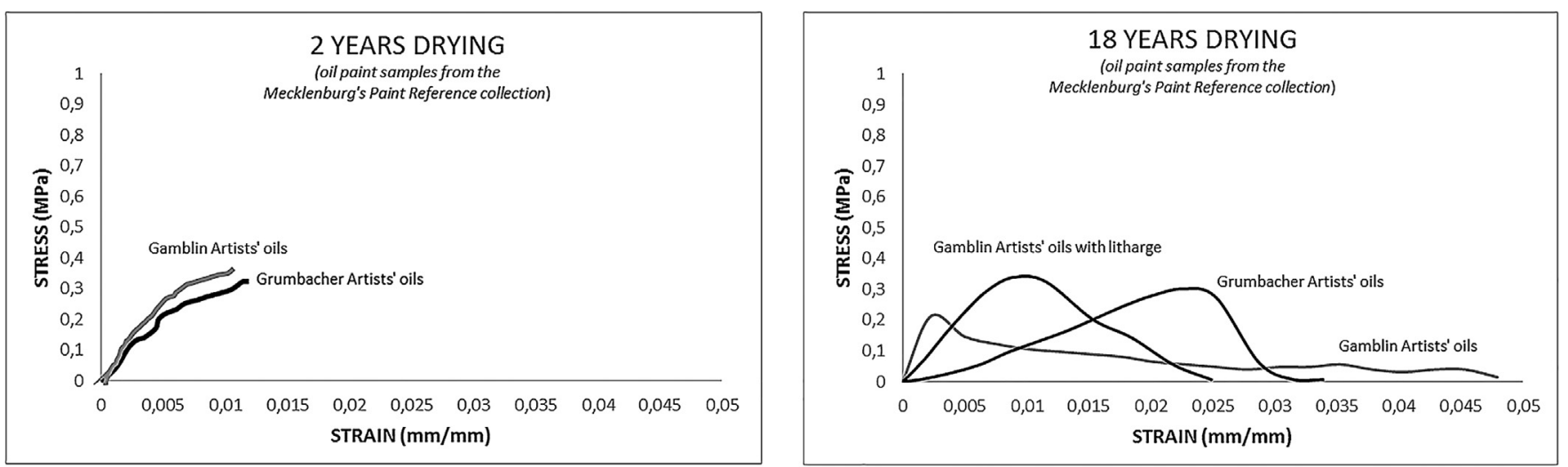

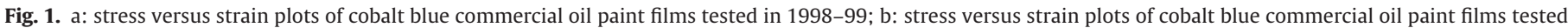
in 2017.

FTIR-ATR analyses were performed with a Thermo Nicolet Nexus 670 FTIR spectrophotometer combined with a Smart Orbit Single Reflection Diamond ATR accessory, from 4000 to $400 \mathrm{~cm}^{-1}$ for 128 scans with $4 \mathrm{~cm}^{-1}$ resolution. Spectra were elaborated with Omnic 8.0. in the range $1800-1600 \mathrm{~cm}^{-1}$, the second derivative was calculated.

A Trace GC 2000 instrument with a capillary Supelco Equity-5 column, $30 \mathrm{~m}, 0.25 \mathrm{~mm}, 0.5 \mu \mathrm{m}$ interfaced with a Trace MS 2000 was employed for GC-MS analysis. The temperature programme was set from $80^{\circ} \mathrm{C}$ to $300^{\circ} \mathrm{C} 10^{\circ} \mathrm{C} / \mathrm{min}$. The MS was run in Full Scan mode (m/z 40-600), 1.9 scans/s. The transfer line was at $280^{\circ} \mathrm{C}$ and the source temperature was $150^{\circ} \mathrm{C}$. Electron ionisation energy was $70 \mathrm{eV}$. Quantitative GC-MS analysis was performed using nonadecanoic acid as the internal standard. The oil samples were transestherified using (trifluoromethylphenyl) trimethylammonium hydroxide, overnight reaction as described in $[2,16]$. The molar ratios amongst the most significant fatty acids were calculated: palmitic to stearic acid $(\mathrm{P} / \mathrm{S})$, azelaic to palmitic acid $(\mathrm{A} / \mathrm{P})$, oleic to stearic acid $(\mathrm{O} / \mathrm{S})$ ratios were considered and compared to previous results obtained by the authors on 20th century artists oil paints as described in $[2,3,25]$.

\section{Results and discussion}

Table 4 reports a detailed summary of the multi-analytical chemical-physical results on the studied paint films.

Although the selected paints have similar commercial names and were sold as "cobalt blue" paints, their surface appearance is quite different (see pictures in Figs. 1 and 2) and their formulations are rather complex.

The analytical results can be briefly summarised highlighting:

- the use of different binding media (for oil paints: ranging from traditional linseed oil to less drying oils, such as safflower and sunflower oils; for alkyds: alkyd resin and drying oils); 
a)

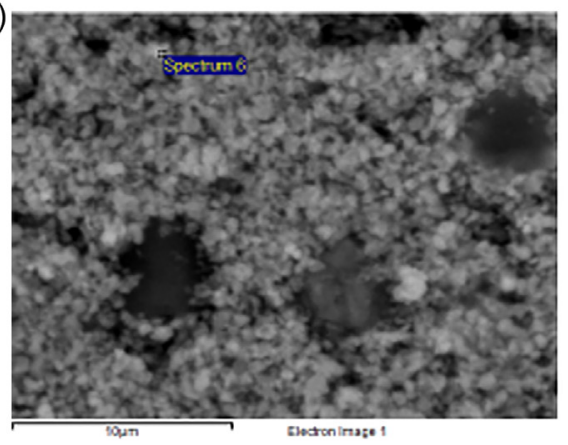

b)

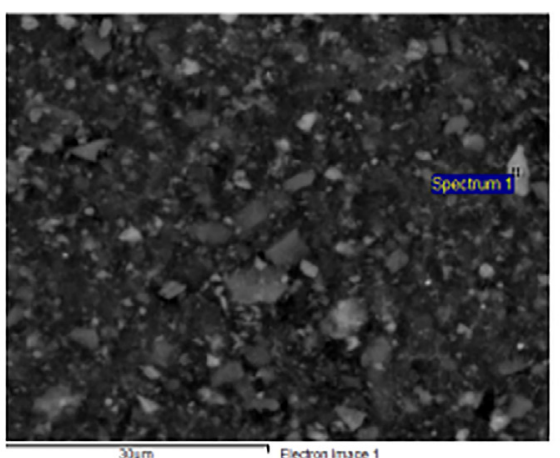

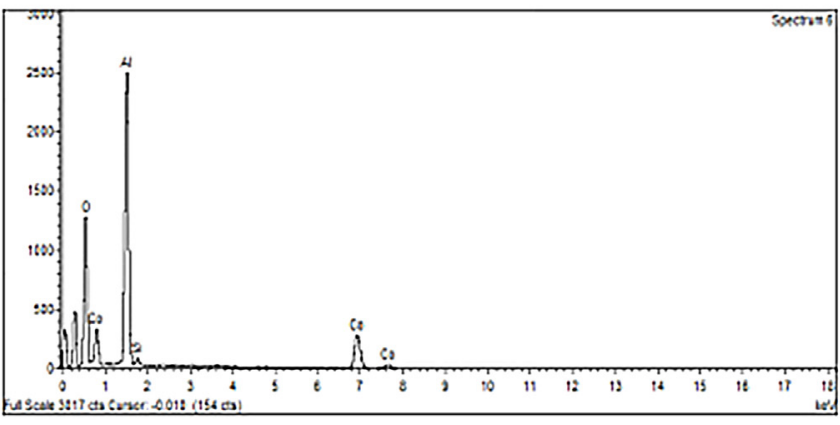

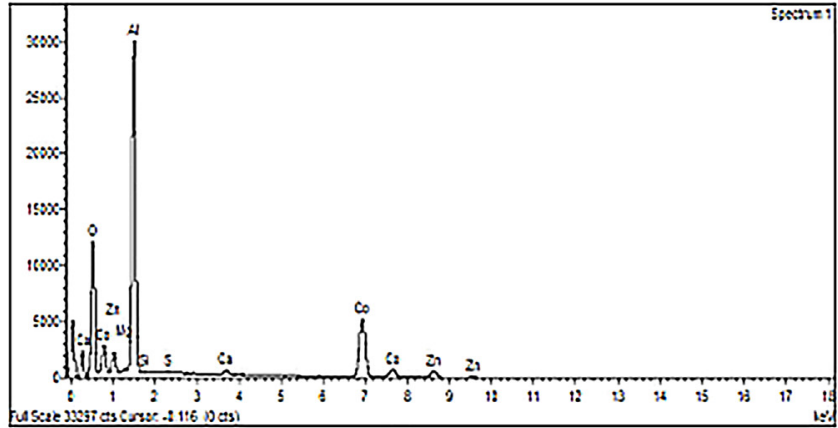

Fig. 2. a: chromatogram GR-CB-ARLO; b: chromatogram WN-CB-ALK.

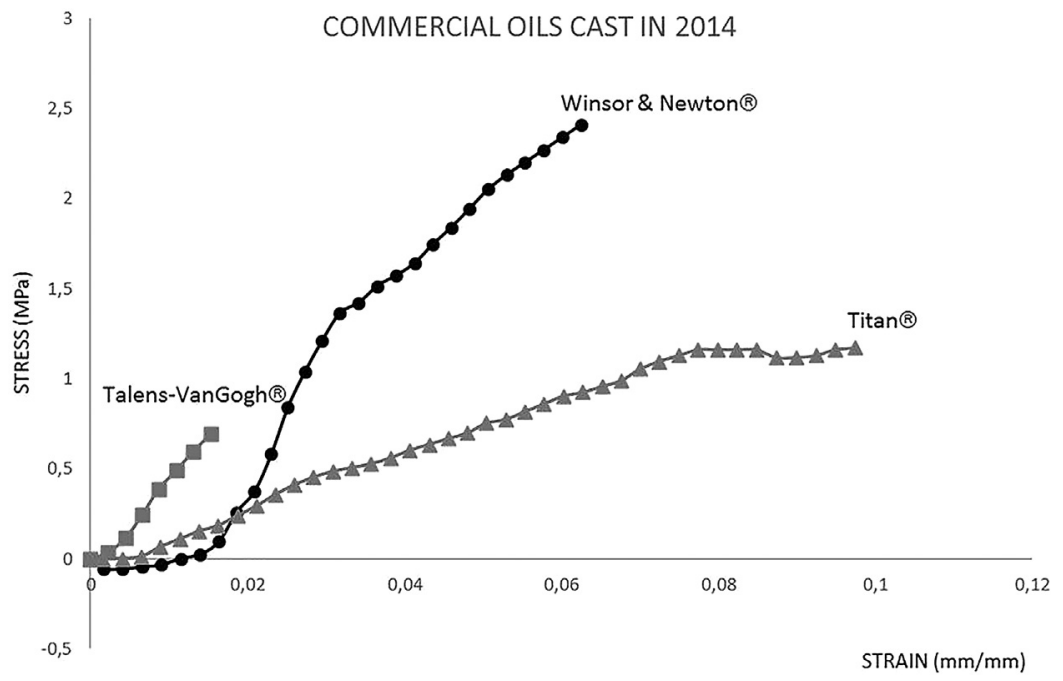

Fig. 3. Stress versus strain plots of cobalt blue commercial oil paint films cast in 2014 .

- the addition of inorganic and organic pigments to cobalt blue (such as, for instance, ultramarine blue);

- variable percentages of cobalt;

- the presence of extenders and fillers (such as silicates, calcium sulphate, calcium carbonate, dolomite, etc) dispersing agents (such as metal stearates) and other additives (zinc white).

These findings are consistent with the literature about the analysis of modern and contemporary oil-based paints [1-6,15,19].

As concerns the mechanical measurements, not all the paints were tested. Free films were obtained and tested for all samples except for WN-CB (from the 1990s), Talens-Van Gogh and Maimeri (casted in 2014), because it was not possible to detach the paint films from the polyester substrate.

\subsection{Commercial cobalt blue oil paint films}

Fig. 1a presents the stress-strain curves of GR-CB-ARLO and GACB-ARLO paint samples tested by Mecklenburg et al. after 2 years drying [21]. Both Gamblin and Grumbacher paint films present a weak behaviour with very little strength (less than $0.5 \mathrm{MPa}$ ) and $1 \%$ elongation. The low modulus of elasticity observed could indicate a very young (and not completely cross-linked) oil paint film. Fig. 1b shows the mechanical properties of the same paint films after 18 years drying. Surprisingly, they present the same weak behaviour (less than $0.5 \mathrm{MPa}$ ) depicted in Fig. 1a, together with a significant increase in plasticity (from $0.01 \mathrm{~mm} / \mathrm{mm}$ strain observed in 2001 in both samples to $0.04 \mathrm{~mm} / \mathrm{mm}$ and $0.05 \mathrm{~mm} / \mathrm{mm}$ observed in 2017 for Grumbacher and Gamblin, respectively). In both cases, one could expect stiffer and stronger paint films as a consequence 


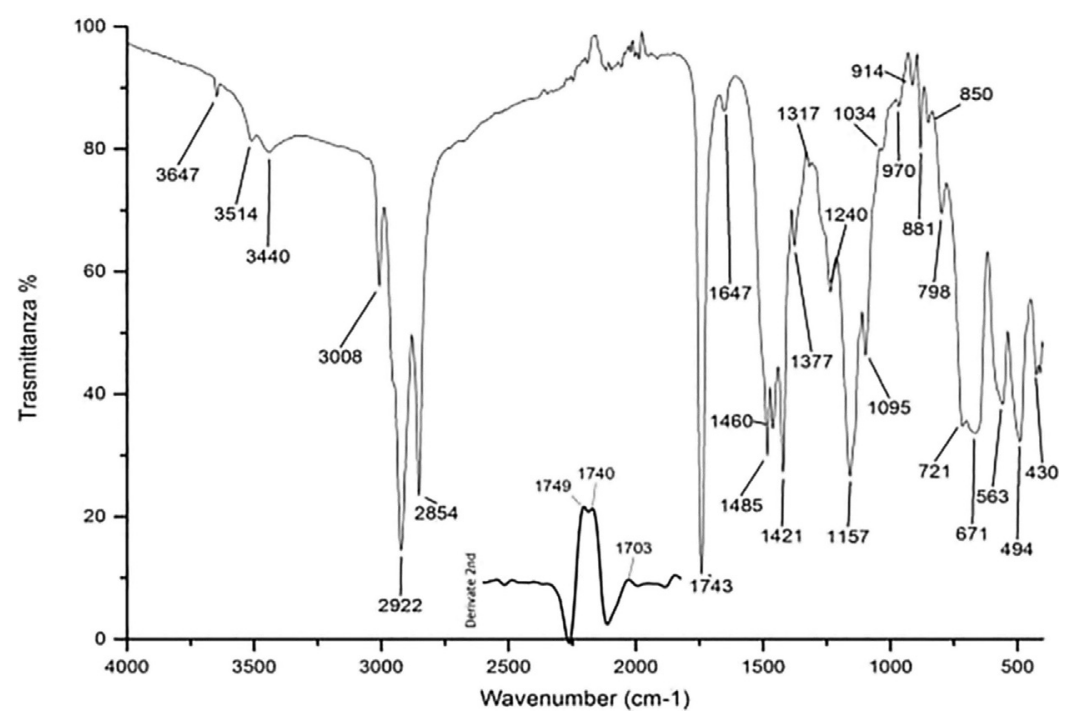

Fig. 4. Second derivative of the $1800-1600 \mathrm{~cm}^{-1}$ range (sample WN-CB 178).

of longer drying times and therefore a more crosslinked structure, as usually observed in oil paints. Nevertheless, the increased plasticity could be a consequence of the early hydrolysis experienced by the oil paint film [26].

GA-CB-LIT (a Gamblin paint sample containing litharge, PbO, and cast in 1999) was tested for comparison purpose. Previous studies $[12,13,20]$ shown that, even in small amounts (up to $1.6 \%$ ), PbO is an effective drier for oils (linseed, poppy seed, walnut and safflower) traditionally used by artists. It can speed up the drying of oil-based paints and induce significant differences in their mechanical properties. Nevertheless, this does not necessarily mean that it contributes to form better paint films.

In this study, it was interesting to compare cobalt blue and litharge siccative properties. Even if cobalt has already relevant siccative properties, litharge seems to enhance them. Results have shown that the presence of a low amount of litharge promotes the thorough drying of GA-CB-LIT in the bulk but also in the surface. This could also be related to its wrinkled appearance.

Considering the lipidic binding media, it is interesting that Gamblin and Grumbacher were found to contain alkali refined linseed oil (i.e. a raw linseed oil treated with alkali to reduce the free acidity by formation of water-soluble salts, which are subsequently removed by washing). As previously stated, different drying speeds can be observed as a function of the refining process of linseed oil: the refining in alkali or acidic mediums makes linseed oil dry two times faster than cold pressed oils [12,13]. Moreover, the low P/S ratios of Grumbacher and Gamblin ( 0.8 and 1.1, respectively) could be related to the addition of dispersing agents (such as aluminium stearates), as reported in previous studies $[2,3,15,19]$. These metal soaps are generally used to raise the acidity of the oil and to wet pigments, in particular when added to formulations containing alkali refined linseed oils with a reduced amount of free fatty acids [27]. From a mechanical point of view, their presence could be responsible for the increase in plasticity observed with age in samples GR-CB-ARLO and GA-CB-ARLO [1,2,27].

Another consideration arises observing the A/P ratios for GR-CBARLO, GA-CB-ARLO and GA-CB-LIT $(2.5,2.3$, and 4.2, respectively). High $\mathrm{A} / \mathrm{P}$ ratios (higher in GA-CB-LIT due to the catalytic action of lead ions) are usually related to a high degree of chain scission which could also explain a less crosslinked (and therefore more flexible) paint film [28] (see Fig. 2).

Fig. 3 represents the stress-strain curves of three cobalt blue Titan, W\&N and Talens-VanGogh commercial oil paint films cast in 2014 (corresponding to samples TI-CB, WN-CB178 and TA-CB, respectively), and tested after 3 years drying. As highlighted by the curves, the samples show significant differences in their mechanical properties.

The first aspect that deserves attention is the UTS: W\&N is about more than two times stronger $(2.5 \mathrm{MPa})$ than Titan and TalensVanGogh (1 MPa and 0.7 MPa, respectively).

A possible explanation is linked to the GC-MS results on the organic fraction. Talens-VanGogh, Maimeri and Titan paint films were found to be bound in linseed oil, since their $\mathrm{P} / \mathrm{S}$ ratios are consistent with linseed oil values (Table 4). However, the P/S ratio for W\&N paint is 2.5 and, together with a relevant presence of specific fatty acids (arachidicbehenic acids) in its composition, may indicate the presence of safflower and/or sunflower oil in the paint formulation $[3,15]$. A similar $\mathrm{P} / \mathrm{S}$ ratio $(2.8)$ was found in $\mathrm{WN}-\mathrm{CB}$, cast in 1998, which is in accordance with the information provided in W\&N's data sheets in which a mixture of linseed and safflower oils is declared.

The use of slow drying oils such as safflower and/or sunflower oil in paints decrease the speed of drying. A relatively young paint film containing safflower and/or sunflower oils would be expected to remain flexible (even plastic) during a long period of time. Nevertheless, the stepper curve obtained for samples WN-CB178 deserves further attention. As reported in FTIR-ATR spectrum of Fig. 4, the presence of dolomite was detected which is an indication of the presence of fillers. A high pigment volume concentration could have counteracted the action of the slow drying oil by speeding up its drying as well as the stiffening of the paint film [15].

Talens-VanGogh, being apparently stiffer, appeared to be significantly weaker than W\&N and Titan films from 2014 (Fig. 3). As previously observed in GR-CB and GA-CB films, the $\mathrm{P} / \mathrm{S}$ ratio $<1$ of Talens-VanGogh could suggest the addition of metal stearates, visible also by FTIR. EDX elemental analysis has highlighted the presence of Zinc, likely in the form of zinc oxide (or zinc white), which is frequently added in commercial paint formulations as a whitener/extender. In all three mock-ups, it is relevant to note that, having been drying for only 3 years, their UTS is significantly higher (2.5 MPa for W\&N and $1 \mathrm{MPa}$ in the case of Titan) than the UTS observed in the samples belonging to the Mecklenburg's collection tested after 18 years $(\sim 0.35 \mathrm{MPa})$. The presence of $\mathrm{ZnO}$ white makes paint films become brittle in their early drying stages but also to cause delamination problems. Zinc, being another metal compound 
a)
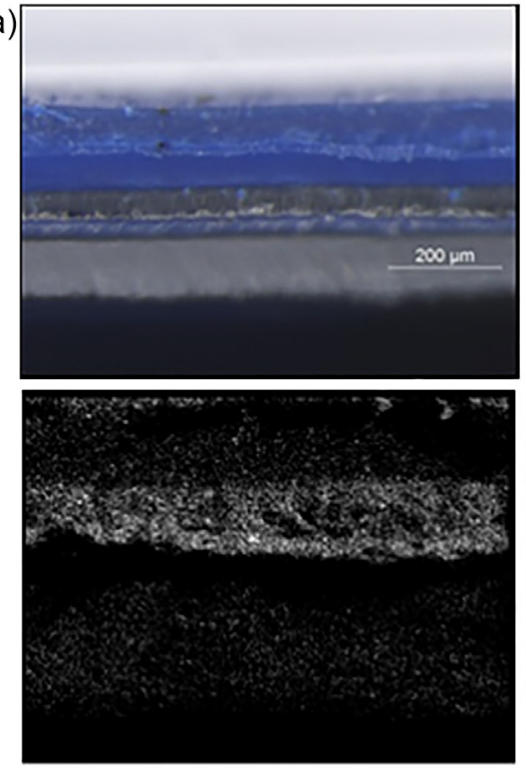

Al Ka1

b)
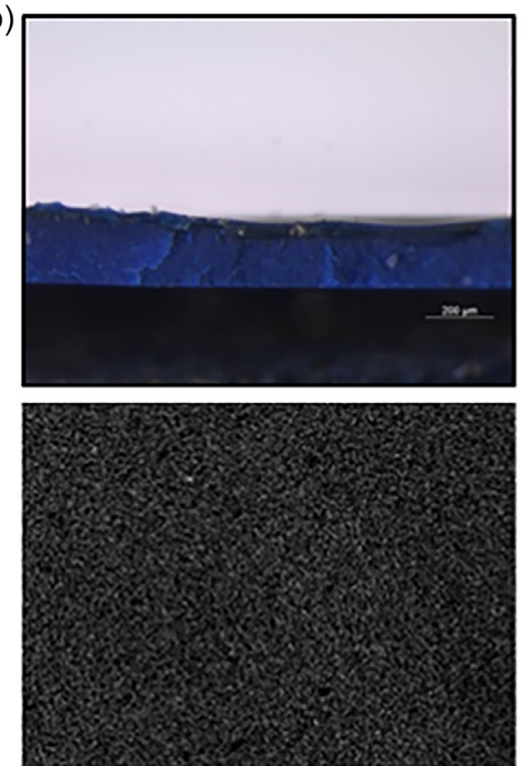

A Ka1
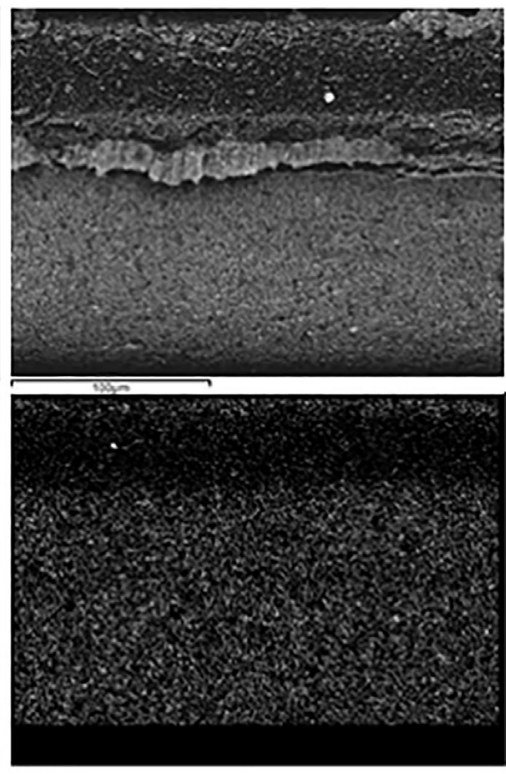

CoKa1
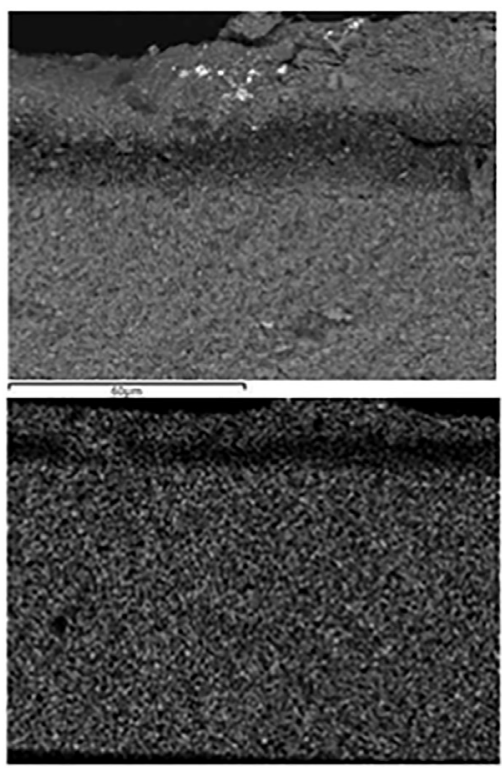

Coka1

Fig. 5. a: SEM MA-CB: Co and Al particles distribution; b: SEM GA-CB-ARLO: Co and Al particles distribution.

existing in more than one valence state, speeds up the drying rate of drying oils [29-31].

Regarding the role of cobalt blue pigment in the mechanical performance of the selected oil paint films, there are several aspects to be highlighted. Cobalt Blue was identified in all the commercial samples studied (Table 4) as indicated by the typical IR absorptions of cobalt oxide (c.a. 640,543 and a sidekick at $800 \mathrm{~cm}^{-1}$ ) and of aluminium oxide $\left(486 \mathrm{~cm}^{-1}\right)$ and the VIS-Reflectance spectra showing the typical weak inflection at $480 \mathrm{~nm}$ and three sub-bands at 548 , 583 , and $627 \mathrm{~nm}[32,33]$. In the case of Titan, ultramarine blue was detected as well. This is interesting since it is known that - as it happens with cobalt -, ultramarine has a very high oil index pigment and therefore a high oil content. Cobalt blue pigment presents a very high oil-absorption index, which means that it needs a significant amount of oil to form a uniform paint film [16]. This can be related to the activity of metal cations. Since the catalytic activity during oxidative polymerization falls with a decreasing proportional content of cobalt cations dissolved in the organic phase, it can be expected that cobalt oil paint films, being highly absorbent, will experience slow drying times. The presence of a higher oil content could explain the lower E-modulus and higher flexibility observed for TI-CB [16,34].

SEM analysis evidenced a uniform distribution of cobalt and aluminium particles except for MA-CB, where cobalt particles seem to be more concentrated on the bulk, whereas aluminium particles tend to concentrate on surface (Fig. 5). EDX analysis revealed an extremely variable \% Co probably due to the presence of other compounds in the different paint matrixes (Table 4). In addition, both the size and shape of pigment particles as well as the chemical composition of other minerals present in the matrix might contribute to make the catalytic activity decrease. Šimůnková et al. suggest that the rate of polymerization of the drying oil increases with PVC and it is different for the various types of cobalt blue pigment [35]. Other studies refer to the influence of PVC in the mechanical properties of paint films. For example, a high PVC will make paint films be stiffer and have a lower failure strain. Nevertheless, above the 

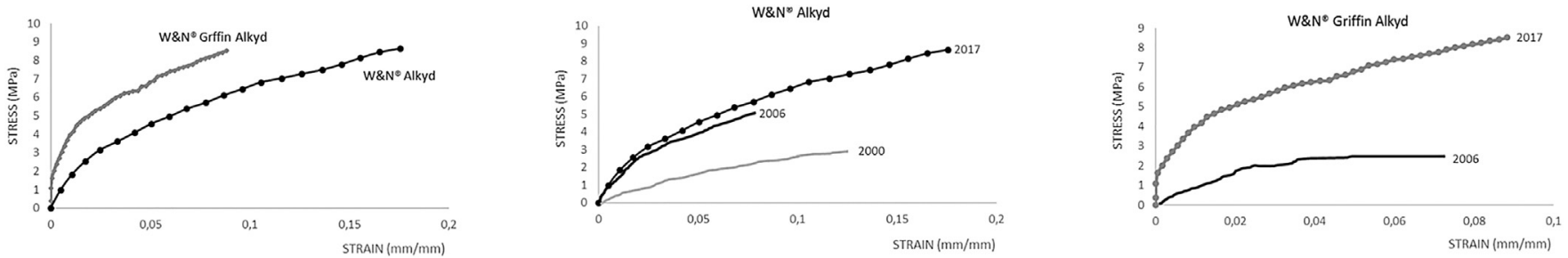

Fig. 6. a, b, c: stress versus strain plots of cobalt blue commercial alkyd paint films cast in the late 1990's in different drying stages.
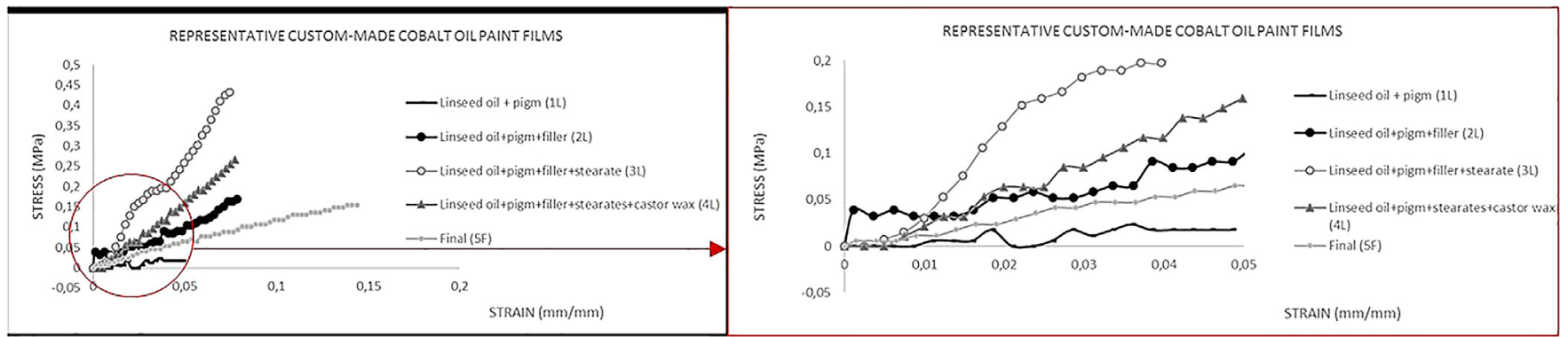

Fig. 7. Stress versus strain plots of cobalt blue representative custom-made samples.

Critical Pigment Volume Concentration (CPVC), paint films present a low UTS and failure strain but also a poorly bound pigment [36].

\subsection{Commercial cobalt blue alkyd paints films}

Fig. 6a shows the stress-strain curve of W\&N Alkyd Paint and W\&N Griffin Artists'Alkyd Paint (samples WN-ALK and WN-GR-ALK respectively). Cobalt blue alkyd commercial paints tested in this study are more than ten times stronger, stiffer and elongable than commercial cobalt blue oil paints studied after similar drying times.

Alkyd resins are oil-modified alkyd polyesters or, in other words, artificial drying oils that autoxidize and cross-link depending on their degree of unsaturation [5]. According to the GC-MS results, WN-ALK and WN-GR-ALK contain an alkyd resin modified with linseed oil ( $\mathrm{P} / \mathrm{S}=1.6$ and 1.7 , respectively) and hydrogenated castor oil (also known as castor wax) whose marker - 12-hydroxy stearic acid-, was found in both samples [37]. This wax-like material is often added in oil and alkyd-based paint formulations as a stabilizer or rheology additive to prevent oil separation. The presence of waxy materials as stabilizers is known to increase the wettability of the pigment, as a sort of lubricant, increasing oil absorption $[34,35]$. This could explain the high failure strains observed.

Another relevant aspect is the presence of zinc white in both samples (Table 4). As explained previously, zinc ions are sufficient mobile to interact with cobalt and make the paint film become stiffer in a relatively short period of time [14] as Fig. 6a shows. Fig. $6 \mathrm{~b}$ shows the evolving mechanical properties of WN-ALK in different drying stages (after 2, 8 and 18 years). As it can be observed, the sample becomes stiffer and stronger over time and the same applies for WN-GR-ALK (Fig. 6c).

\subsection{Cobalt blue custom-made paint film reconstructions}

A series of representative mock-up samples were prepared in 2014 (Table 3) aiming at gaining an insight into the role each component plays in the mechanical behaviour of a given oil paint film.

Fig. 7a shows the stress-strain curve of the samples $1 \mathrm{~L}, 2 \mathrm{~L}, 3 \mathrm{~L}, 4 \mathrm{~L}$ and $5 \mathrm{~F}$. In the case of $1 \mathrm{~L}, 2 \mathrm{~L}, 3 \mathrm{~L}$ and $4 \mathrm{~L}$ a detailed look at the elastic region of the different stress-strain curves (Fig. $7 \mathrm{~b}$ ) suggest that $2 \mathrm{~L}$ (the sample with the addition of fillers) is the one with a highEModulus. Even if further tests are needed, this increased stiffness could be related to the fact that the addition of fillers (until a certain CVPV) can modify the PVC of the sample formulated with binder and pigment alone as previously explained.

The addition of stearates (3L) shows a curve with a lower EModulus but that reaches a higher UTS than $2 \mathrm{~L}$, whereas $4 \mathrm{~L}$ (sample containing stearates and $10 \%$ castor wax) is more flexible and presents a lower UTS. It can be observed that $2 \mathrm{~L}, 3 \mathrm{~L}, 4 \mathrm{~L}$ present a considerable deformation at break $(0.15 \mathrm{~mm} / \mathrm{mm})$ if compared with commercial oils tested $(0.05 \mathrm{~mm} / \mathrm{mm})$ and could be due to the fact that the representative samples were fresh oils tested shortly after being cast ( 2 years).

Finally, Sample 5F containing a mixture of CPLO, safflower and sunflower oils is twice elongable than the samples made with only CPLO ( $1 \mathrm{~L}$ to $4 \mathrm{~L}$ ), which is in agreement with previous studies about the use of slow drying oils and therefore the significant plasticity they usually present. This is particularly true if we compare $5 \mathrm{~F}$ with $4 \mathrm{~L}$, which was prepared with a similar amount of pigment, fillers and additives but with a different medium

\section{Conclusions and future perspectives}

This study provides an insight into the mechanical properties of selected cobalt blue paint formulations. Besides the complexity of defining the composition of modern oil and alkyd paint films labelled as "cobalt blue" paints, there are often encountered synergic circumstances that make it difficult to discriminate a single cause for the changes observed in their mechanical performance over time.

For the oil paint films studied, the influence of the different degree of unsaturation of the lipid binders in the mechanical properties of the paint films was shown. Alkyd paints tested were found to be more than ten times stronger and stiffer than the commercial cobalt blue oil paints studied after similar drying times. They also showed a higher elongation at break. This could be governed by the different binding medium but also by the presence of zinc white, that might have contributed to make the alkyd paint film become stiffer in a relatively short period of time.

The amount of cobalt present mostly affects its degree of dispersion in the lipid binder. This points out that a given compound - either acting as pigment or as a siccative - might induce different mechanical properties in the resulting paint film as the 
stress-strain curves obtained from the examined commercial formulations have shown. It has been observed that a greater quantity of pigment absorbed by the drying oil corresponds to a more important catalytic activity (i.e. a greater drying speed with a consequent increase in the stiffness of the paint film). Furthermore, it was also observed that the addition of other drying agents modifies the activity of cobalt. A significant example is litharge that competes with cobalt acting mainly in the bulk of the paint creating a stiffer film that oxidizes faster. Nevertheless, results showed in this paper make it difficult to confirm to what extent the quantity of cobalt influences the condition of the paint films examined.

As observed in the custom-made cobalt oil paint films studied, additives have shown to play a significant role in the mechanical properties of the paint films studied. For example, a significant amount of dispersing agents was found in the studied commercial formulations probably added to slow down the speed of the drying process.

Further research is needed to gain an insight into the relationship between UTS the drying of the films (for example through the evaluation of the $\mathrm{A} / \mathrm{P}$ ratios) and into the role of additives in the mechanical performance of commercial paint films.

\section{Acknowledgements}

The authors are deeply indebted to Dr. M.F. Mecklenburg (Museum Conservation Institute-Smithsonian Institution, USA) for providing the samples tested in this study and for the equipment donated. This study was carried out as part of the research project HAR2016-75131-P granted by the Minsterio de Economia, Industria y Competitividad (MICINN/FEDER) and was also possible thanks to the financial support from Ca' Foscari University of Venice (Premio Giovani Ricercatori).

\section{References}

[1] TJS Learner, Analysis of Modern Paints, The Chemistry of Modern Paint, Oil, The Getty Conservation Institute, Los Ángeles, USA, 2004.

[2] FC Izzo, 20th Century Artists' Oil Paints: A Chemical-Physical Survey, Dottorato di ricerca in Scienze Chimiche, Università Ca'Foscari, Venezia, 2010.

[3] FC Izzo, K van den B, H van Keulen, B Ferriani, E Zendri, Modern oil paints - formulations, organic additives and degradation: some case studies, in: KJ van den Berg, A Burnstock, M de Keijzer, J Krueger, T Learner, A de Tagle, G Heydenreich (Eds.), Issues in Contemporary Oil Paint, Springer International Publishing, Switzerland, 2016, pp. 75-104.

[4] JJ Boon, FG Hoogland, J Van Der Horst, Mass spectrometry of modern paints, in: Modern paints uncovered: proceedings from The Modern Paints Uncovered Symposium organized by the Getty Conservation Institute, Tate, and the National Gallery of Art, Tate Modern, London, 2007.

[5] R Ploeger, The Characterization and Stability of Artists' Alkyd Paints (PhD Dissertation), University of Torino, 2008.

[6] Keune, Katrien, and Gwendolyn Boevé-Jones. It's surreal: zinc oxide degradation and misperceptions in Salvador Dalí's Couple with Clouds in their Heads, 1936. Issues in Contemporary Oil Paint. Springer, Cham, 2014. 283-294.

[7] S Keck, Mechanical alteration of the paint film, Stud. Conserv. 14.1 (1969)9-30.

[8] A Karpowicz, In-plane deformations of films of size on paintings in the glass transition region, Stud. Conserv. 34.2 (1989) 67-74.

[9] A Karpowicz, A study on development of cracks on paintings, J. Am. Inst. Conserv. 29.2 (1990) 169-180.

[10] D. Rogala, S. Lake, C. Maines, M. Mecklenburg, Condition problems related to zinc oxide underlayers: examination of selected abstract expressionist paintings from the collection of the Hirshhorn Museum and sculpture garden, Smithsonian Institution, Journal of the American Institute for Conservation 49 (2) (2010) 96-113.

[11] D Erhardt, CS Tumosa, MF Mecklenburg, Natural and accelerated thermal aging of oil paint films, Stud. Conserv. 45 (sup. 1) (2000) 65-69.
[12] M. F. Mecklenburg, C. S. Tumosa, D. Erhardt, The changing mechanical properties of aging oil paints, in P. B. Vandiver, J. L. Mass, A. Murray, eds. Materials Issues in Art and Archaeology VII: Symposium held November 30-December 3, 2004, Boston, Massachusetts, USA, Warrendale, Pennsylvania. Materials Research Society, (2005), pp. 13-24.

[13] D. Erhardt, C.S. Tumosa, M.F. Mecklenburg, Long-term chemical and physica process in oil paint films, Stud. Conserv. 50 (2005) 143-150.

[14] M. F. Mecklenburg, C. S. Tumosa, E. Vicenzi, The influence of pigments and ion migration on the durability of drying oil and alkyd paints. In: Mecklenburg, M., Elena Charola, A. \& Koestler, R. J., Eds. New insights into the cleaning of paintings, (2012) Universidad Politécnica de Valencia. Smithsonian Contributions to Museum Conservation.

[15] L. Fuster-López, F.C. Izzo, M. Piovesan, D.J. Yusa Marco, L. Sperni, E. Zendri, Study of the chemical composition and the mechanical behavior of 20th century commercial artists' oil paints containing manganese-based pigments, Microchem. J. 124 (2014) 962-973.

[16] A. Roy, Cobalt blue, in artists' pigments, in B. Berrie (Ed.), A handbook of their history and characteristics, Vol. 4, Oxford University Press, National Gallery of Art, Washington (2007), pp. 151-178.

[17] J. Plesters, Ultramarine Blue, Natural and Artificial, in A. Roy (ed), Artists' Pigments, A Handbook of their History and Characteristics, vol. 2, National Gallery of Art, Washington, Archetype Publications, London, (1993), 37-61.

[18] F Delamare, The Discovery of Thenard's (Cobalt) Blue, A Time for Scientists, in Blue Pigments: 5000 Years of Art and Industry, cap.6, Archetype Publications 2013, pp. 195-206

[19] FC Izzo, B Ferriani, KJ Van den Berg, H Van Keulen, E Zendri, 20th century artists oil paints: the case of the Olii by Lucio Fontana, J. Cult. Herit. 15 (5) (2014) 557-563.

[20] MF Mecklenburg, CS Tumosa, An introduction into the mechanical behavior of paintings under rapid loading conditions, in: MF Mecklenburg (Ed.), Art in Transit: Studies in the Transport of Paintings, London, National Gallery of Art, Washington DC, 1991, pp. 137-171.

[21] MF Mecklenburg, L Fuster-López, Estudio de las Propiedades Mecánicas y Dimensionales de los Materiales Pictóricos, Universidad Politécnica de Valencia, 2013.

[22] S Mansour, Use of ZAF and PAP matrix correction models for the determination of carbon in steels by electron probe microanalysis, X-Ray Spectrom. 18 (6) (1989) 263-266.

[23] L Osete-Cortina, MT Doménech-Carbó, A Doménech, DJ Yusá-Marco, H Ahmadi, Multimethod analysis of Iranian Ilkhanate ceramics from the Takht-e Soleyman palace, Anal. Bioanal. Chem. 397 (2010) 319-329.

[24] M Piovesan, Pitture ad olio a base di "Terra d'ombra naturale" e "Terra d'ombra bruciata": studio delle formulazioni industriali e del comportamento termico e meccanico (MA thesis), Univ. Ca Foscari, Venezia, 2014.

[25] KR Sutherland, Derivatisation using m(trifluoromthyl)phenyltrimethylammonium hydroxide of organic materials in artworks for analysis by gas chromatography-mass spectrometry: unusual reaction products with alcohols, J. Chromatogr. A 1149 (2007) 30-37.

[26] JDJ van den Berg, Analytical Chemical Studies on Traditional Linseed Oil Paints, AMOLF FOM, Geboren te Woerden, MOLART Reports, 2002.

[27] CS Tumosa, A brief history of aluminum stearate as a component of paint, WAAC Newslett. 23 (3) (2001) 10-11.

[28] MC Corbeil, K Helwig, J Poulin, Jean Paul Riopelle: The Artist's Materials, Ed. Getty Conservation Institute, Los Ángeles, USA, 2011, pp. 55-56.

[29] CS Tumosa, MF Mecklenburg, The influence of lead ions on the drying of oils Rev. Conserv. 6 (2005).

[30] G Osmond, Zinc white: a review of zinc oxide pigment properties and implications for stability in oil-based paintings, AICCM Bull. 33 (1) (2012) 20-29.

[31] G Osmond, Zinc White and the Influence of Paint Composition for Stability in Oil Based Media. Issues in Contemporary Oil Paint, Springer, Cham, 2014, pp. 263-281.

[32] M Bacci, M Picollo, Non-destructive spectroscopic detection of Cobalt (II) in paintings and glasses, Stud. Conserv. 41 (1996) 136-144.

[33] M Bacci, D Magrinia, M Picollo, A study of the blue colors used by Telemaco Signorini (1835-1901), J. Cult. Herit. 10 (2) (2009) 275-280.

[34] R Mayer, The Artist's Handbook of Materials and Techniques, Ed. Viking, New York, USA, 1991.

[35] E Šimůnková, J Brothánková-Bucifalová, J Zelinger, The influence of cobalt blue pigments on the drying of linseed oil, Stud. Conserv. 30 (4) (1985) 161-166.

[36] E. Hagan, M. Charalambides, T.J.S. Learner, A. Murray, C. Young, Factors Affecting the Mechanical Proprieties of Modern Paints, in T.S.J. Learner P. Smithen, J.W. Krueger, R. Shilling, Modern Paints Uncovered, Proceedings from the Modern Paints Uncovered Symposium organized by the Getty Conservation Institute, Tate and the National Gallery of Art, London, (2006) pp. 227-235.

[37] JS Mills, R White, The Organic Chemistry of Museum Objects, Series in Conservation \& Museology, second ed., Butterworth-Heinemann, London, 1994. 